\title{
Effects of allogeneic mouse adipose-derived mesenchymal stem cell-microporous sheep acellular dermal matrix on healing of wound with full-thickness skin defect in mouse and the related mechanism
}

\author{
Shengjun C, Lingfeng W*, Te B, Xue F, Fang L, Chunguang H \\ Burn Research Institute of Inner Mongolia, Department of Burns, the Third Affiliated Hospital of Inner Mongolia Medical \\ University, Baotou, Inner Mongolia, China
}

Received: April 22, 2019

DOI: $10.5430 /$ dcc.v6n2p26
Accepted: May 30, 2019

Online Published: June 10, 2019

\begin{abstract}
Objective: To explore the effects of allogeneic mouse adipose-derived mesenchymal stem cell (ADSC)-microporous sheep acellular dermal matrix (ADM) on wound healing of full-thickness skin defect in mice and the related mechanism.

Methods: One Kunming mouse was sacrificed by cervical dislocation to collect adipose tissue from the inguinal region. Mouse ADSCs were isolated from the adipose tissue and cultured in vitro. Cells in the third passage were identified by cell adipogenic and osteogenic differentiation. The expressions of CD34, CD73, CD90, and CD105 were analyzed by flow cytometer. After one sheep was sacrificed with the skin of its back cut off, microporous sheep ADM was prepared by using acellular processing and freeze-thaw method. A round and full-thickness skin defect wound, with a diameter of $12 \mathrm{~mm}$, was made on the back of each of 36 Kunming mice. The wounds were covered by microporous sheep ADM. The mice were divided into ADSC group and control group with 18 mice in each group according to the random number table method after surgery. A volume of $0.2 \mathrm{ml}$ of DMEM/F12 culture medium containing $1 \times 10^{6}$ ADSCs was injected between microporous sheep ADM and the wound of each mouse in ADSC group, while $0.2 \mathrm{ml}$ of DMEM/F12 culture medium was injected between microporous sheep ADM and the wound of each mouse in control group. At post-surgery day (PSD) 12 and 17, the wound healing rate in each group was calculated respectively; wound vascularization in 2 groups of mice was observed under the reverse irradiation of back light; and the granulation tissue in the wound in ADSC group was observed by means of hematoxylin-eosin staining. At PSD 7, the thickness of the granulation tissue in the wound was measured in each group of mice. At PSD 12 and 17, the immunohistochemical method was used to detect the expression of VEGF in each group of mice. The number of samples was 6 in each group at each time point in the above experiments. The data obtained were processed with t-test and factorial design ANOVA.

Results: (1) After 7 days of adipogenic induction, red lipid droplets were observed in the cytoplasm with oil red O staining. After 21 days of osteogenic induction, black calcium deposition was observed in the medium stained with silver nitrate. The expression levels of CD73, CD90, CD 105 and CD34 in cells were 97.82\%, 99.32\%, 97.35\% and 5.88\% respectively. The cells were identified as ADSCs. (2) The wound healing rates of ADSC group at PSD 12 and 17 [(78 \pm 6$) \%$, (98 \pm 3$) \%]$ were significantly higher than those of control group at PSD 12 and 17 [(60 \pm 9$) \%,(90 \pm 4) \%, t=4.26,4.46, p<.01]$. (3) At PSD 7 , no vessels obviously grew into the center of the wound in both groups of mice, while the granulation tissue already covered the wound in ADSC group. At PSD 12, the wound in ADSC group was more well-perfused than control group. At PSD 17, it was observed that large vessels were crossing through the whole wound in ADSC group, while large vessels were observed without
\end{abstract}

*Correspondence: Lingfeng W; Email: wlf7413@vip.sina.com; Address: Burn Research Institute of Inner Mongolia, Department of Burns, the Third Affiliated Hospital of Inner Mongolia Medical University, Baotou, Inner Mongolia 014010, China. 
crossing through the whole wound in control group. (4) In ADSC group, at PSD 7, the wound was covered with thin granulation tissue, and the granulation tissue was obviously thickened at PSD 12. At PSD 17, the granulation tissue was covered by epidermis. At PSD 7, the thickness of the granulation tissue in the wound in ADSC group [ $0.62 \pm 0.05) \mathrm{mm}]$ was significantly greater than that in control group $[(0.31 \pm 0.04) \mathrm{mm}, t=12.27, p<.01]$. (5) At PSD 12 and 17, the expression levels of VEGF in the wound in ADSC group $\left[(80.7 \pm 2.2),(102.8 \pm 2.6) / \mathrm{mm}^{2}\right]$ were significantly than those in control group $[(59.5 \pm 2.4),(81.5 \pm$ $\left.2.6) / \mathrm{mm}^{2}, t=15.95,14.14, p<.01\right]$.

Conclusions: Allogeneic mouse ADSC-microporous sheep ADM can promote angiogenesis and the growth of granulation tissue in the wound with full-thickness skin defect in mice, thus accelerating wound healing. The mechanism is probably related with the increase in the expression of VEGF.

Key Words: Wounds and injuries, Mesenchymal stem cell transplantation, Biological dressings, Wound healing, Adiposederived mesenchymal stem cells, Acellular dermal matrix

\section{INTRODUCTION}

Burns and surgical operations often result in extensive fullthickness skin defect, and it is needed to perform an extensive skin renewal for the sake of treatment. ADM is easy to store, and it can cover skin defect and restore skin barrier function in a short term. Furthermore, its three-dimensional structure provides a space frame for the growth of tissue cells. It can promote epidermal adhesion and proliferation, and it is considered to be the best skin substitute, which has a remarkable effect in the treatment of skin defect. ${ }^{[1,2]}$ Adipose-derived mesenchymal stem cell (ADSC) is a type of mesenchymal stem cell with multi-directional differentiation potential, it can differentiate into different lineages of cells. It has been extensively studied in the fields of regenerative medicine and tissue engineering. ${ }^{[3]}$ A research shows that ADSC can promote the proliferation of vascular endothelial cells and $\mathrm{Fb}$ through intercellular direct contact and paracrine action to accelerate wound healing. ${ }^{[4,5]}$ In this research, mouse ADSC-ADM is applied to the treatment of full-thickness skin defect in mice, simultaneously, the synergistic effect of ADSC-ADM and its mechanism are detected to provide a theoretical basis for the further preparation of novel dressings containing recombinational stem cells.

\section{MATERIALS AND METHODS}

\subsection{Animals, main reagents and the source of instru- ments}

One healthy and clean male sheep, 3 months old, weighed $20 \mathrm{~kg} ; 37$ healthy male Kunming mice with no special pathogens, 10 weeks old, each weighed 20-22 g. All animals were purchased from Experimental Center of Inner Mongolia University, Certification No.: SCXK (M2016-0001). DMEM/F12 medium and trypsin solution were purchased from HyClone (USA); fluorescein isothiocyanate (FITC) labeled mouse anti-human CD34 monoclonal antibody, phycoerythrin (PE) labeled mouse anti-human CD73 monoclonal antibody, FITC labeled mouse anti-human CD90 monoclonal antibody and PE labeled mouse anti-human CD105 monoclonal antibody were bought from Ebioscience (USA); oil red $\mathrm{O}$ and silver nitrate were made by Sigma (USA); Triton X-100 was purchased from Amresco (USA); rabbit antihuman vascular endothelial growth factor (VEGF) primary antibody, goat anti-rabbit IgG secondary antibody and VEGF IHC kits were bought from Wuhan Boster Biological Technology Co., Ltd.. HZQ-F160 constant-temperature shaking incubator was purchased from Taicang Huamei Biochemical Instrument Factory, HF-90 carbon dioxide incubator was bought from Hongkong Heal Force Bio-meditech Holdings Limited, FAC-SCalibur flow cytometer was made by BD (USA), FD-1B-80 freeze dryer was bought from Beijing Boyikang Experimental Instrument Co., Ltd., JSZ5B stereoscopic microscope was made by Shanghai Hengqin Instrument \& Equipment Co., Ltd., and DMIL LED optical microscope was purchased from Leica (Germany).

\subsection{Isolation, cultivation and identification of mouse ADSC}

One mouse was selected and acclimated for 1 week. Adipose tissues in the bilateral inguinal regions were collected under the aseptic condition. Collagenase digestion, density gradient centrifugation and adherence method were applied to the isolation, cultivation and purification of cells. Cells in a good state in the third passage were selected and then identified by cell adipogenic and osteogenic differentiation (After adipogenic induction, lipid droplets stained by oil red $\mathrm{O}$ showed red; after osteogenic induction, lipid droplets stained by silver nitrate showed the appearance of black calcium deposition). The flow cytometer was used to detect the expression levels of CD34, CD73, CD90 and CD105 for follow-up experiments.

\subsection{Preparation of microporous sheep ADM}

Microporous sheep ADM was prepared according to the method mentioned in the literature. ${ }^{[6]}$ One sheep was sac- 
rificed with the skin on the back cut off: the mechanical shaving was given and drum type dermatome was used to cut the dermis reversely, in order to prepare $0.2-0.3 \mathrm{~mm}$ (thickness) of skin tissues. The skin tissue was trimmed to the size of $5 \mathrm{~cm} \times 5 \mathrm{~cm}$, and then immersed with $1 \mathrm{~g} / \mathrm{L}$ of benzalkonium bromide for $30 \mathrm{~min}$. Later, the tissue was rinsed with PBS for 5 times and placed into $2.5 \mathrm{~g} / \mathrm{L}$ of human trypsin solution for 24-hour processing. After rinsed with PBS for 5 times, the tissue was immersed with $0.5 \%$ Triton $\mathrm{X}-100(\mathrm{~V} / \mathrm{V})$ for 24-hour continuous shaking. The tissue was rinsed with PBS for another 5 times, and then sheep $\mathrm{ADM}$ was placed in the freeze dryer for freeze drying, with microporous sheep ADM acquired consequently.

\subsection{Modeling, group processing and specimen collection} Each of 36 mice was injected with $100 \mathrm{~g} / \mathrm{L}$ of chloral hydrate at a dose of $0.03 \mathrm{ml} / \mathrm{kg}$ by abdominal cavity for anesthesia, with the hair on the back removed, $75 \%$ ethanol (V/V) for disinfection. Surgical scissors was used to cut off a round full-thickness skin with a diameter of $12 \mathrm{~mm}$, and $12 \mathrm{~mm}$ (inner diameter) of rigid plastic ring was used to tackle the boundary of the wound, to guarantee that the area of the wound in each mouse was identical. Microporus sheep ADM was placed into the rigid plastic ring, with nylon sutures used for interrupted suture (see Figure 1). After surgery, the mice were divided into ADSC group and control group with 18 mice in each group according to the random number table method. A volume of $0.2 \mathrm{ml}$ of DMEM/F12 culture medium containing $1 \times 10^{6}$ ADSCs was injected between microporous sheep ADM and the wound of each mouse in ADSC group, while $0.2 \mathrm{ml}$ of DMEM/F12 culture medium was injected between microporous sheep ADM and the wound of each mouse in control group. All mice in these two groups were fed separately.
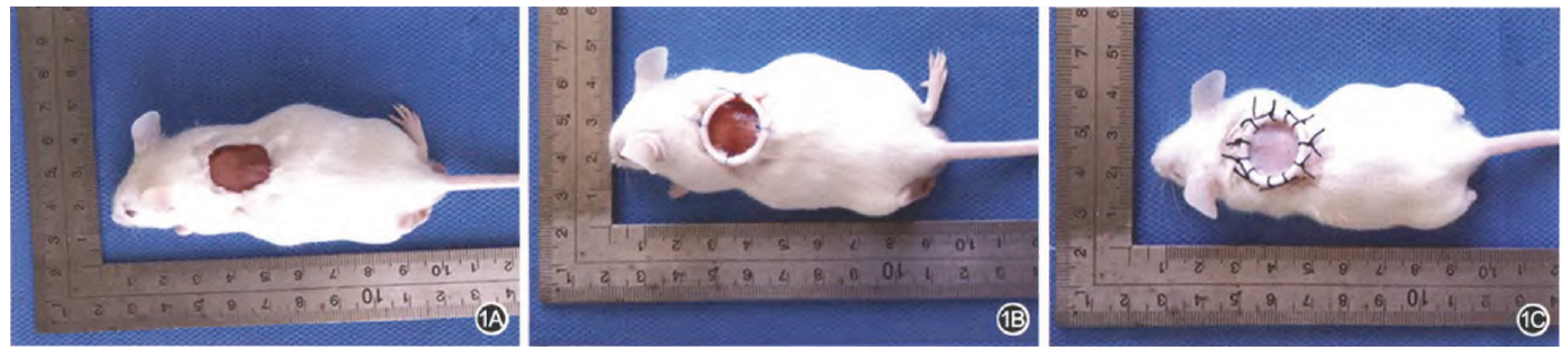

Figure 1. Modeling and coverage of wound with full-thickness skin defect in mice

A. Cutting off a round full-thickness skin with a diameter of $12 \mathrm{~mm}$; B. Rigid plastic ring used to tackle the boundary of the wound;

C. Microporus sheep ADM was used to cover the wound within the rigid plastic ring, with nylon sutures used for interrupted suture.

\subsection{Indicator observation}

\subsubsection{Wound healing rate}

6 mice were selected at PSD 7, PSD 12 and PSD 17 respectively, and then sacrificed by use of a closed chamber full of $\mathrm{CO} 2$, sheep ADM removed. A digital camera was used to take photographs for recording. The complete epithelization of the wound was considered as the healing standard. Image $\mathrm{J} 1.43$ image processing software (USA National Institutes of Health) was used to measure the residual area of the wound and calculate wound healing rates at PSD 12 and PSD 17: wound healing rate $=($ original area of the wound - residual area of the wound) / original area of the wound $\times 100 \%$.

\subsubsection{Wound vascularization}

The whole wound tissue (deep to the layer of fascia) in each mouse in 2 groups at 3 time points mentioned in 2.5.1 was removed and observed with the stereoscopic microscope $(40 \times)$ for wound vascularization under the reverse irradiation of back light.

\subsubsection{Thickness of the granulation tissue in the wound} At PSD 7, 12 and 17, the wound tissue taken from each mouse in ADSC group was fixed with $10 \% \mathrm{NBF}$ (V/V), and part of the tissue was embedded with paraffin and resected (thickness $5 \mu \mathrm{m}$ ). After deparaffinage and dehydration, the tissue was stained conventionally with HE. The granulation tissue was observed under the optical microscope $(50 \times)$. In addition, the thickness of the granulation tissues was measured for 2 groups of mice: the granulation tissue with a width of $3 \mathrm{~mm}$ on the center of the wound was selected to measure its central width and edge widths to calculate the mean value, which was considered as the thickness of the granulation tissue.

\subsubsection{Expression of VEGF in the wound}

At PSD 12 and 17, the immunohistochemical method was used to detect the expression of VEGF in the residual wound tissue fixed with NBF solution in two groups of mice. After high-pressure heat mediated antigen retrieval, the paraffinembedded section (thickness $5 \mu \mathrm{m}$ ) was rinsed, resected, added with rabbit anti-human VEGF primary antibody (dilu- 
tion ratio $1: 100)$, then washed and added with goat anti-rabbit IgG secondary antibody (dilution ratio 1:100), incubated at $37^{\circ} \mathrm{C}$ for $30 \mathrm{~min}$, developed with DAB, re-stained with hematoxylin and observed under the optical microscope $(400 \times)$ for the positive expression of VEGF (brownish yellow) in the wound tissue. 6 visual fields were selected from each section, and the cells with the positive expression of VEGF in each field were counted and averaged. The result was represented by the number of cells with positive expression of VEGF per square milimeter.

\subsection{Statistical treatment}

SPSS 17.0 statistical software was applied to statistical processing. The measurement data fitted to normal distribution, and data with homogeneity were represented by $\bar{X} \pm$ s. The overall comparison was made by use of factorial design ANOVA, and the comparison between two group at each time point was made by use of $t$-test. The difference $p<.05$ was of statistical significance.

\section{Results}

\subsection{Cell identification}

After 7 days of adipogenic induction, red lipid droplets were observed in the cytoplasm with oil red $\mathrm{O}$ staining (see Figure 2). After 21 days of osteogenic induction, black calcium deposition was observed in the medium stained with silver nitrate (see Figure 3). The identification results obtained by flow cytometer showed that, the expression levels of characteristic phenotypes (of mesenchymal stem cells) CD73, CD90 and CD105 were 97.82\%, 99.32\% and 97.35\% respectively, all showing a high expression. The expression of HSC marker CD34 was 5.88\%.The cells were identified as ADSCs.

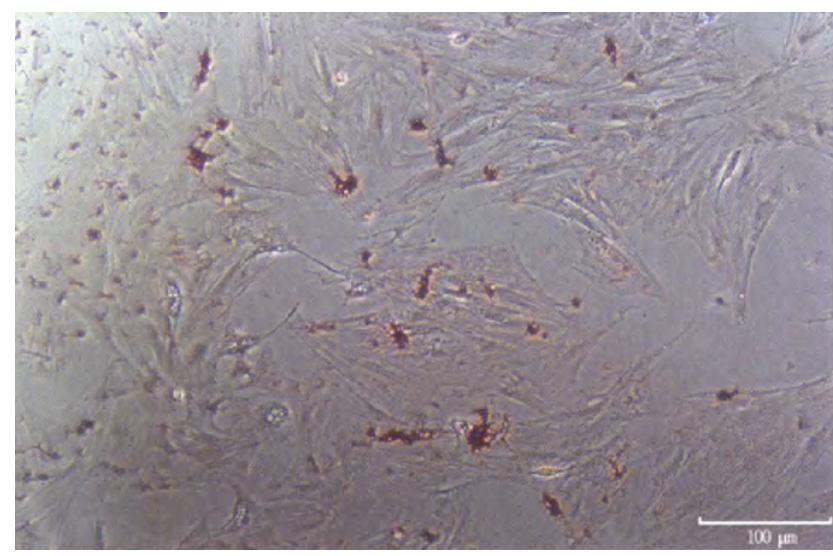

Figure 2. After 7 days of adipogenic induction to cultured ADSCs in the third passage, red lipid droplets were observed (oil red $\mathrm{O} \times 200$, scale: $100 \mu \mathrm{m}$ )

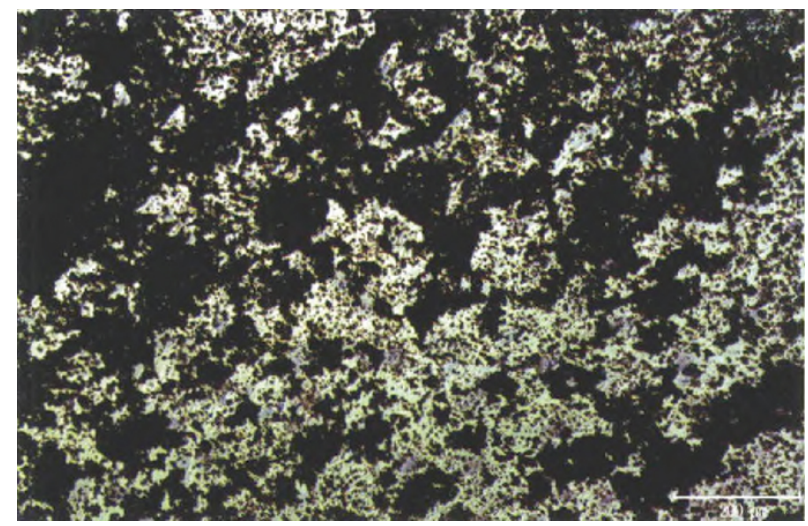

Figure 3. After 21 days of osteogenic induction to cultured ADSCs in the third passage, black calcium deposition was observed (silver nitrate $\times 200$, scale: $100 \mu \mathrm{m}$ )

\subsection{Wound healing rate}

The wound healing rates of ADSC group at PSD 12 and 17 were significantly higher than those of control group at PSD 12 and $17(p<.01)$. See Table 1 for details.

Table 1. The comparison of wound healing rates at PSD 12 and 17 between two groups of mice with full-thickness skin defect $(\%, \bar{X} \pm \mathrm{s})$

\begin{tabular}{llll}
\hline Group & Number of Mice & $\mathbf{1 2 ~ d}$ & $\mathbf{1 7} \mathbf{d}$ \\
\hline ADSC Group & 12 & $78 \pm 6$ & $98 \pm 3$ \\
Control Group & 12 & $60 \pm 9$ & $90 \pm 4$ \\
$t$ Value & & 4.26 & 4.46 \\
$p$ Value & & .002 & .001 \\
\hline
\end{tabular}

Note. Main effect of treatment factor, $F=32.06, p<.001$; Main effect of time factor, $F=121.73, p<.001$; Mutual effect of treatment and time factors, $F=4.74, p=.042$

Table 2. The comparison in expression levels of VEGF at PSD 12 and 17 between two groups of mice with full-thickness skin defect $\left(\mathrm{n} / \mathrm{mm}^{2}, \bar{X} \pm \mathrm{s}\right)$

\begin{tabular}{llll}
\hline Group & Number of Mice & $\mathbf{1 2 ~ d}$ & $\mathbf{1 7 ~ d}$ \\
\hline ADSC Group & 12 & $80.7 \pm 2.2$ & $102.8 \pm 2.6$ \\
Control Group & 12 & $59.5 \pm 2.4$ & $81.5 \pm 2.6$ \\
t Value & & 15.95 & 14.14 \\
$p$ Value & & $<.001$ & $<.001$ \\
\hline
\end{tabular}

Note. Main effect of treatment factor, $F=447.22, p<.001$; Main effect of time factor, $F=482.98, p<.001$; Mutual effect of treatment and time factors, $F=0.01, p=.935$

\subsection{Wound vascularization}

At PSD 7, no vessels obviously grew into the center of the wound in both groups of mice, while the granulation tissue already covered the wound in ADSC group. At PSD 12, the wound in ADSC group was more well-perfused than control group. At PSD 17, it was observed that large vessels were crossing through the whole wound in ADSC group, while large vessels were observed without crossing through the whole wound in control group. See Figure 4 for details. 


\subsection{Thickness of the granulation tissue in the wound}

In ADSC group, at PSD 7, the wound was covered with thin granulation tissue, and the granulation tissue was obviously thickened at PSD 12. At PSD 17, the granulation tissue was covered by epidermis. See Figure 5 for details. At PSD 7 , the thickness of the granulation tissue in the wound in ADSC group $[(0.62 \pm 0.05) \mathrm{mm}]$ was significantly greater than that in control group $[(0.31 \pm 0.04) \mathrm{mm}, t=12.27, p<.01]$.

\subsection{Expression of VEGF in the wound}

The wound healing rates of ADSC group at PSD 12 and 17 were significantly higher than those of control group at PSD 12 and 17 (see Table 2).
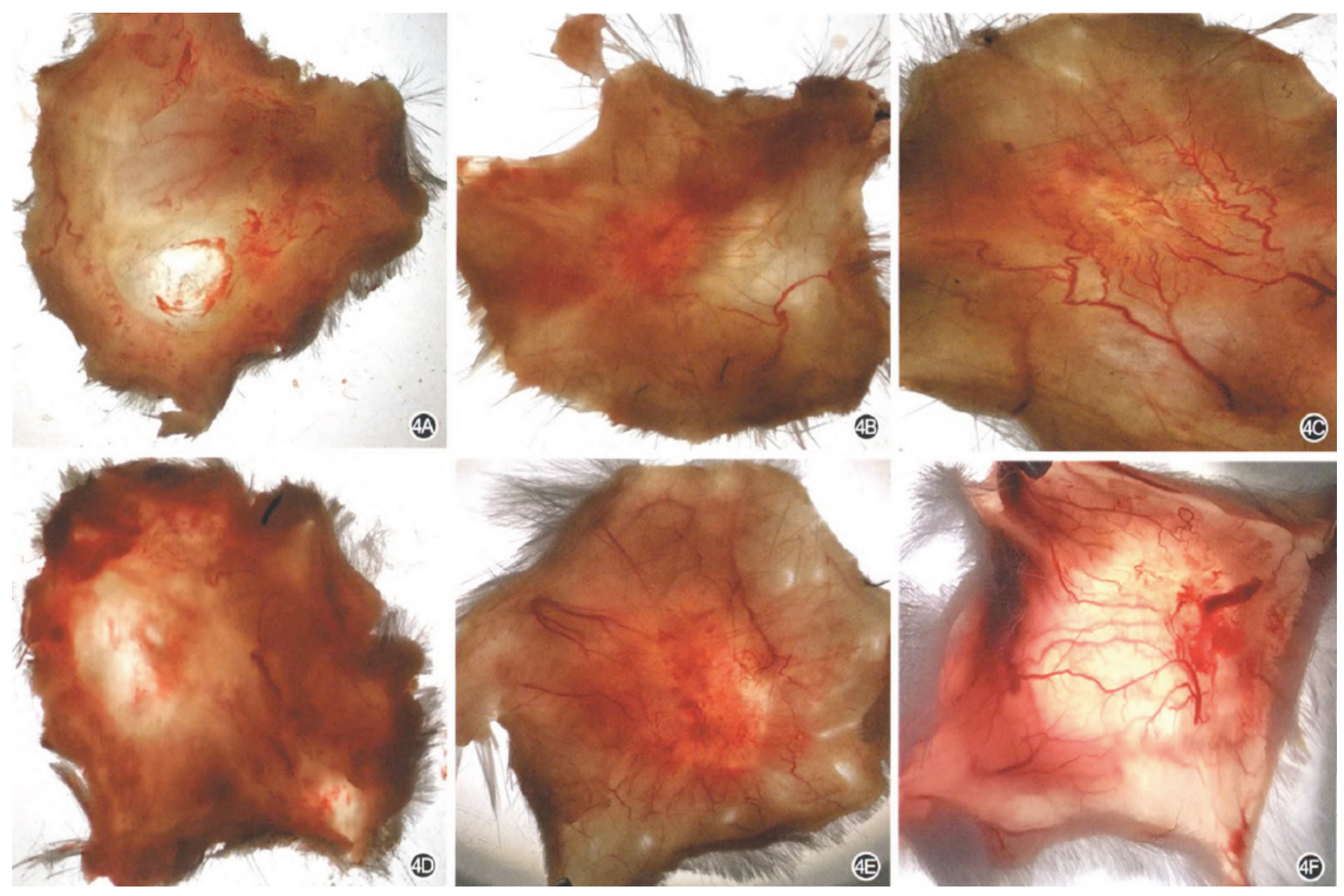

Figure 4. Wound vascularization of full-thickness skin defect in 2 groups of mice (stereoscopic microscope $\times 4$ ) A. At PSD 7, no vessels obviously grew into the center of the wound; B. At PSD 12, a few vessels obviously grew into the center of the wound; C. At PSD 17, it was observed that a large number of vessels grew into the center of the wound, but there were no large vessels crossing through the whole wound; D. At PSD 7, no vessels obviously grew into the center of the wound, while the granulation tissue already covered the wound in ADSC group; E. At PSD 12, a large number of vessels obviously grew into the center of the wound in ADSC group; F. At PSD 17, it was observed that large vessels were crossing through the whole wound in ADSC group.

The lucent region is the center of the wound; ADSCs are adipose-derived mesenchymal stem cells.
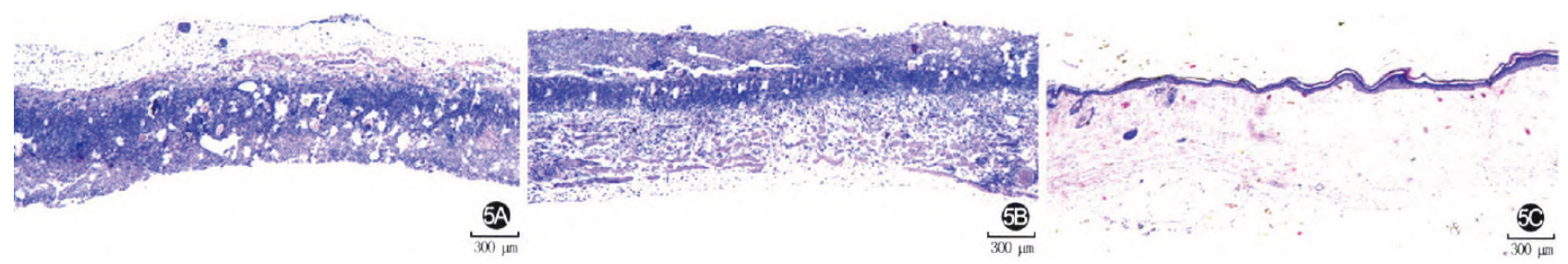

Figure 5. The thickness of granulation tissue in mouse full-thickness skin defect in ADSC group at each time point after surgery $(\mathrm{HE} \times 50$, scale: $300 \mu \mathrm{m})$

A. At PSD 7, the granulation tissue was thin; B. At PSD 12, the granulation tissue was obviously thickened; C. At PSD 17, the granulation tissue was covered by epidermis. 


\section{Discussion}

The objective of tissue engineering and regenerative medicine is to construct biological substitutes to recover the normal function of impaired and lesion tissues. ${ }^{[7]}$ Various tissue engineering skins used for wound repair spring up clinically, such as ADM, Integra ${ }^{\circledR}$ and Biobrane ${ }^{\circledR} \cdot{ }^{[8]}$ In addition, some cells, which have an effect on promoting healing, can also be used to repair wounds, such as autologous active skin cells, stem cells ${ }^{[9,10]}$ et al. Tissue engineering is focused on the usage of biological materials for the temporary coverage of wounds, but it has a limited function of promoting healing due to lack of skin cells. Regenerative medicine puts particular emphasis on the usage of cells which can promote wound healing to regulate healing reaction. However, in the practical application, cell therapy is limited by poor cell viability. ${ }^{[11]}$ Therefore, the author assumes to use microporus ADM as a support to uniformly deliver ADSC onto the wound, so that ADM has a barrier function and ADSC can retain its effect on promoting healing. Some scholars have inoculated ADSCs to mouse ADM. ${ }^{[12]}$ Unlike the abovementioned experiment, the author chooses microporus ADM as the covering for mouse wound. It is because it is found in the experiment that sheep skin is thin, soft and ductile, and it is of certain air permeability and ventilation and better moisture retention. ${ }^{[6]}$ Cells, vessels and cutaneous appendages are eliminated from sheep skin after acellular processing, so that immune response can be relieved effectively after transplantation. After deep hypothermic freeze-thaw processing, the dermis becomes softer, with apertures distributed uniformly. The retained fibrous skeleton and collagens correspond closely to biological properties of skin, and have an effect on inducing the growth of host cells. ${ }^{[12-14]}$ In addition, the preparation and preservation method is easy to use.
There are a large number of ADSCs used in this experiment exsiting in the body. It is practicable to acquire ADSCs directly from adipose tissues abandoned during surgery, which is easy to operate.

This research shows that angiogenesis in ADSC group is faster than that in control group at PSD 7, with a thicker granulation tissue. ADSC group is still faster in angiogenesis than control group both at PSD 12 and PSD 17, and much earlier to establish a vascular network which crosses through the whole surface of wound. The wound healing rate in ADSC group is higher than that in control group. This result indicates that ADSC - microporus ADM accelerates the formation of the granulation tissue and the speed of angiogenesis in the wound, improve the tissue microenvironment and promote wound healing. The mechanism of stem cell therapy is mainly paracrine action. ${ }^{[15]}$ Stem cells activate specific signaling pathway and accelerate the repair of tissue damage by secreting cytokines, especially VEGF, plays an important role. The immunohistochemical results in this research show that the wound healing rates of ADSC group at PSD 12 and 17 are significantly higher than those of control group at PSD 12 and 17. It is indicated that the expression of VEGF is increased in the wound tissue, which can promote wound vascularization. Reverse vascular transillumination result also confirms this assumption. Therefore, allogeneic mouse ADSC-microporous sheep ADM can promote angiogenesis and the growth of granulation tissue in the wound with full-thickness skin defect in mice, thus accelerating wound healing. The mechanism is probably related with the increase in the expression of VEGF.

\section{CONFlicts OF InTEREST Disclosure}

The authors declare they have no conflicts of interest.

\section{REFERENCES}

[1] Zhang Y, Zeng Y, Xin G, et al. Biological function evaluation and effects of laser micro-pore burn-denatured acellular derma matrix. Burns. 2018; 44(2): 350-358. PMid:28823469. https://doi.org/ $10.1016 / j$. burns .2017 .07 .009

[2] Li X, Meng X, Wang X, et al. Human acellular dermal matrix allograft: a randomized, controlled human trial for the long-term evaluation of patients with extensive burns. Burns. 2015; 41(4): 689-699. PMid:25687834. https://doi.org/10.1016/j . burns.2014.1 2.007

[3] Shengjun C, Lingfeng W, Te B. Advances in the research of basic study and clinical application of adipose-derived mesenchymal stem cells. Chinese Journal of Burns. 2017; 33(3): 184-189.

[4] Yongming Y, He Y, Zemin Z, et al. Effects of rabbit adipose-derived mesenchymal stem cells on the healing of skin deep partial-thickness scald wound of rabbit. Chinese Journal of Burns. 2016; 32(7): 402407.

[5] Sukho P, Cohen A, Hesselink JW, et al. Adipose tissue-derived stem cell sheet application for tissue healing in vivo: a systematic review. Tissue Eng Part B Rev. 2017; 24(1): 37-52. PMid:28665192. https://doi.org/10.1089/ten.teb.2017.0142

[6] Chunguang H, Lingfeng W, Xiaguang D, et al. Study on the material of human umbilical cord mesenchymal stem cells-microporous sheep acellular dermal matrix. Chinese Journal of Injury Repair and Wound Healing (Electronic Edition). 2016; 11(1): 24-28. https : //doi.org/10.11569/wcjd.v24.i3.362

[7] Atala A. Engineering organs. Curr Opin Biotechnol. 2009; 20(5): 575-592. PMid:19896823. https://doi.org/10.1016/j.copb io. 2009.10.003

[8] Lamy J, Yassine AH, Gourari A, et al. The role of skin substitutes in the surgical treatment of extensive burns covering more than $60 \%$ of 
total body surface area. A review of patients over a 10-year period at the Tours University Hospital. Ann Chir Plast Esthet. 2015; 60(2): 131-139. PMid:24183237. https://doi.org/10.1016/j .anpl as. 2013.09.002

[9] Panxi Y, Jinglong C. Research progress on ReCell ${ }^{\circledR}$ autologous cell in-vitro regeneration technique. Chinese Medical Journal. 2015; 95(12): 955-957.

[10] Holm JS, Toyserkani NM, Sorensen JA. Adipose-derived stem cells for treatment of chronic ulcers: current status. Stem Cell Res Ther. 2018; 9(1): 142. PMid:29764508. https://doi.org/10.1186/ s13287-018-0887-0

[11] Hernon CA, Dawson RA, Freedlander E, et al. Clinical experience using cultured epithelial autografts leads to an alternative methodology for transferring skin cells from the laboratory to the patient. Regen Med. 2006; 1(6): 809-821. PMid:17465762. https: //doi.org/10.2217/17460751.1.6.809
[12] Huang SP, Hsu CC, Chang SC, et al. Adipose-derived stem cells seeded on acellular dermal matrix grafts enhance wound healing in a murine model of a full-thickness defect. Ann Plast Surg. 2012; 69(6): 656-662. PMid:23154338. https ://doi.org/10.1097/SAP.Ob $013 e 318273 f 909$

[13] Jun Z, Zhihui H, Junle J, et al. Animal study on the outcome of sheep acellular dermal matrix in vivo. Chinese Journal of Injury Repair and Wound Healing (Electronic Edition). 2013; 8(3): 295-298.

[14] Chunguang H, Lingfeng W, Xue F, et al. Selective sheep acellular dermis matrix in treatment of deep partial thickness burn rat. Chinese Journal of Injury Repair and Wound Healing (Electronic Edition). 2012; 7(5): 476-481.

[15] Arno Al, Amini-Nik S, Blit PH, et al. Human Wharton's jelly mesenchymal stem cells promote skin wound healing through paracrine signaling. Stem Cell Res Ther. 2014; 5(1): 28 . PMid:24564987. https://doi.org/10.1186/scrt417 